\section{Docencia en violencia y abuso sexual en estudiantes de pregrado de medicina: percepción de los docentes sobre barreras $y$ facilitadores}

\author{
PAMELA ROJAS ${ }^{1, \mathrm{a}}$, CAROLINA RAIN ${ }^{2, \mathrm{~b}}$, \\ VICTORIA CUADRA ${ }^{3, \mathrm{~b}}$, CARLA CASTAÑÓN ${ }^{1, \mathrm{a}}$
}

\section{Teaching medical students about domestic violence and sexual abuse: teachers' perception of barriers and facilitators}

Background: Considering the frequency of sexual abuse and domestic violence $(S A-D V)$ in the population, the likelihood of a medical student meeting patients with this problem is substantial. However, most students do not consider SA-DV in their differential diagnoses. Since 2014, this team has been working in strengthening undergraduate curriculum in SA-DV. It has been a challenging and slow process. Purpose: To identify barriers and facilitators perceived by the undergraduate teaching team in order to suggest modifications in the curriculum in relation to the competencies required for screening and early intervention for victims of $S A-D V$. The conclusions obtained may be useful for other medical schools and universities facing this challenge. Methods: Qualitative study using grounded theory method for the analysis of interviews and focus groups. Results: $S A-D V$ are perceived as a problem that is pertinent for physicians. However, there is a common belief that teaching about SA-DV should be limited to only certain specific specialties. The main barriers are the limited curricular time, the need to prioritize contents and the lack of specific training of the teachers. A key facilitator is the fact that gender violence is on the public agenda today. Conclusions: The identified barriers coincide with those reported in the literature; limited curricular time and prioritization of contents. Although this study does not show the perception of SA-DV as 'not a medical issue' nor as a subject that pertains to other health professionals, there is the perception that teaching clinical approach to SA-DV only pertains to specialists who are directly involved in this topic.

(Rev Med Chile 2018; 146: 927-932)

Key words: Domestic Violence; Sex Offenses; Teaching.

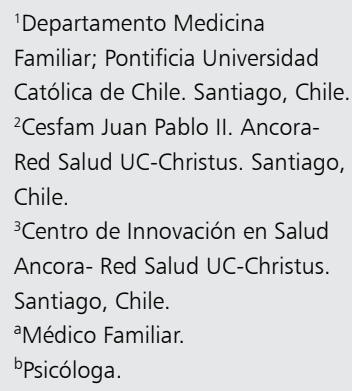

Fuente de apoyo financiero: Este proyecto fue financiado por el Concurso Intradepartamental Departamento Medicina Familiar Pontificia Universidad Católica de Chile. Santiago, Chile.

Los autores declaran no tener conflictos de intereses.

Recibido el 13 de febrero de 2018, aceptado el 19 de julio de 2018.

Correspondencia a:

Pamela Rojas

Departamento Medicina Familiar, Pontificia Universidad Católica de Chile.

Vicuña Mackenna 4686.

Santiago, Chile.

projasgo@uc.cl
L a violencia al interior de las familias (VIF) es una realidad que traspasa países y culturas $^{1}$, y Chile no escapa de este problema. Cifras de la Encuesta Nacional de Victimización por Violencia Intrafamiliar y Delitos Sexuales muestran una prevalencia vida de cualquier tipo de violencia doméstica contra las mujeres chilenas de $35,7 \%{ }^{2}$, mientras que entre los niños las cifras de violencia física grave por parte de uno o ambos padres alcanzaría a $32,8 \%^{2,3}$.

Las cifras sobre violencia sexual en Chile no son más alentadoras. La encuesta de victimización 
ofrece una prevalencia vida de abuso sexual de 7\% en los niños y adolescentes chilenos (cifra que se eleva a $12,8 \%$ en el subgrupo de niñas), y de $15,6 \%$ en mujeres de 15 a 59 años ${ }^{2}$.

La Organización Mundial de la Salud (OMS) recomienda que los sectores de salud y educación desarrollen la capacidad de identificar el abuso sexual infantil ${ }^{4}$. El Instituto de Medicina de Estados Unidos de Norteamérica plantea, por su parte, que el medio más eficaz para mejorar la respuesta a VIF es la educación del médico; propone que la formación en diagnóstico y denuncia sea un aprendizaje prioritario, y ha llamado a que las facultades entreguen estas competencias a sus estudiantes 5 .

En Chile no existe información sistematizada en relación a cuánta formación en violencia intrafamiliar y abuso sexual (VIF-AS) reciben los mé$\operatorname{dicos}^{6}$. Hay consenso en la literatura internacional en que esta formación es reducida, con contenidos escasos en los currículos y con un abordaje por lo general aislado y teórico ${ }^{7-12}$. En la Pontificia Universidad Católica de Chile se han diseñado estrategias para que el estudiante adquiera competencias destinadas a pesquisar y abordar inicialmente un caso de VIF-AS. Si bien los programas están en curso, su instalación ha sido lenta.

Las competencias en VIF-AS deben adquirirse y diversos autores han trabajado en definir qué elementos podrían dificultar la inclusión curricular de VIF-AS en pregrado. Un estudio neozelandés mostró que entre las causas de los médicos para no preguntar por VIF-AS estaban la falta de confianza o experiencia en el abordaje de estas situaciones y una preocupación por empeorar la situación (Miller, 2007) ${ }^{13}$. DeLahunta y Tulsky (1998) identificaron los elementos claves para explicar la resistencia a incorporar VIF-AS en los currículos, destacando el tiempo curricular limitado y cómo este se prioriza; la opinión que la violencia "no es un tema médico", la ambigüedad en las definiciones de maltrato y la falta de soluciones disponibles ${ }^{14}$.

No hay experiencias locales reportadas que permitan definir qué elementos podrían dificultar la inclusión curricular de VIF-AS en el pregrado de medicina. El objetivo de este estudio es indagar en las percepciones del equipo docente de pregrado de la Escuela de Medicina de la Pontificia Universidad Católica de Chile sobre barreras y facilitadores. Acceder a esta información permi- tiría reorientar los esfuerzos realizados y serviría de base para que otras escuelas y universidades se sumen y fortalezcan sus currículos.

\section{Material y Método}

Investigación de carácter cualitativa realizada mediante la utilización de la teoría fundamentada para el análisis de entrevistas y grupos focales. Los participantes fueron seleccionados a través de un muestreo no probabilístico intencionado (Flick, 2004), incorporando informantes claves relacionados con la planificación curricular, e invitados a entrevistas en profundidad o grupos focales, dirigidos por una psicóloga experta en la metodología, audio grabados y transcritos.

Las entrevistas y grupos focales se basaron en una guía de preguntas flexibles. El entrevistador tuvo la libertad de introducir preguntas adicionales para precisar conceptos y obtener más información (Hernández y Fernández, 2006) (Tabla 1), permitiendo generar una discusión en relación al objeto de estudio. Un encuadre adecuado aclaró los alcances y objetivos de la investigación, que indagó solo en el quehacer laboral de los participantes.

Las transcripciones fueron analizadas por 2 investigadores de forma independiente, según los procedimientos de codificación abierta propuestos por la teoría fundamentada, generándose un modelo descriptivo que entregó una visión comprehensiva sobre el tema.

El estudio fue presentado y aprobado por el Comité de Ética de la Escuela de Medicina en la Pontificia Universidad Católica de Chile. Cada participante firmó un consentimiento en el cual se les informó los objetivos de la investigación y su metodología, asegurándoles confidencialidad.

\section{Participantes}

Se realizaron dos entrevistas semiestructuradas y dos grupos focales. Se accedió un total de 10 participantes, seleccionados por el rol que les correspondía en la planificación curricular; pertenecientes a los departamentos de Ginecología, Medicina Interna, Medicina Familiar, Medicina Legal, Pediatría, Salud Pública y Psiquiatría de la Escuela de Medicina de la Pontificia Universidad Católica de Chile. 
Tabla 1. Guión de entrevista para el equipo docente de pregrado

\begin{tabular}{|ll|}
\hline Tópicos & Ejemplos de pregunta \\
$\begin{array}{l}\text { Percepciones acerca de la } \\
\text { integración actual del tema } \\
\text { VIF-AS en pregrado }\end{array}$ & $\begin{array}{l}\text { En la actualidad, ¿cómo integran VIF-AS en curso del cual son responsables? (metodolo- } \\
\text { gía de trabajo, programa del curso, evaluación) }\end{array}$ \\
$\begin{array}{ll}\text { En consultas habituales, ¿cómo observan que se integra VIF-AS como hipótesis diag- } \\
\text { nóstica? }\end{array}$ \\
$\begin{array}{ll}\text { Pédico en VIF-AS } & \text { Hay opiniones que plantean que la violencia no es un tema médico. Desde el rol que cada } \\
\text { Competencias requeridas } & \text { uno tiene en la clínica, ¿qué impresión tienen sobre dicha aseveración? } \\
\text { (considera aspectos } & \text { ¿Cuáles serían las competencias esenciales que debiera tener un docente de pregrado } \\
\text { procedimentales) } & \text { en VIF-AS? } \\
\text { Barreras y facilitadores para la } \\
\text { incorporación al currículo }\end{array}$ & $\begin{array}{l}\text { ¿Qué competencias consideran que son básicas en un egresado de medicina para lograr } \\
\text { un manejo adecuado de situaciones de VIF-AS en una atención clínica? }\end{array}$ \\
& $\begin{array}{l}\text { ¿Qué impresión tienen acerca del conocimiento técnico de los docentes, pensando en la } \\
\text { magnitud y alcance de estos temas? }\end{array}$ \\
\hline $\begin{array}{l}\text { Recursos necesarios para la } \\
\text { incorporación al currículo }\end{array}$ & $\begin{array}{l}\text { Si a ustedes se les considerara para participar en un proceso de cambio que permita la } \\
\text { integración de contenidos sobre VIF-AS atingentes al programa de su curso ¿qué insu- } \\
\text { mos (técnicos u otros) necesitarían para sentirse seguros en la implementación de dicho } \\
\text { cambio? }\end{array}$ \\
\hline
\end{tabular}

\section{Resultados}

A nivel descriptivo, las percepciones expuestas pueden agruparse en 5 áreas centrales: 1 ) abordaje actual; 2) quehacer médico; 3) competencias necesarias; 4) barreras/facilitadores; y 5) recursos necesarios para su abordaje.

\section{Abordaje actual}

Se distinguen 3 aspectos relevantes en el desarrollo actual de VIF-AS en la docencia de pregrado: a) valoración temática; b) desconocimiento del tema; c) abordaje "oportunístico" y conceptual.

"Yo tengo que decir que desconozco si esto se enseña actualmente" (FG1).

"La verdad es que no tengo ninguna información de cómo se enseña o como se mide el tema de violencia intrafamiliar o abuso sexual dentro de pregrado" (E2).

Existe consenso en que VIF-AS es un tema invisible dentro de la formación de pregrado, que aparece de acuerdo con la coyuntura y no como algo planificado y que existiría una falta de herramientas técnicas para su abordaje.
"Me parece insuficiente. Yo creo que es un tema bien invisible (...). No tenemos claridad sobre qué objetivos, metodologías o sistemas de evaluación se estén usando, a diferencia de lo que ocurre con otros temas" (E1).

"En el curso de (especialidad) efectivamente uno de los temas es el abuso físico, maltrato, el abuso sexual, (...) pero ahi no se generan herramientas para enfrentar el abuso, sino que simplemente se analiza en función del impacto que tiene en el desarrollo de la personalidad. No hay un taller de intervención o de qué hacer cuando hay una noticia de estas" (FG2).

"Pudiera salir, pero es alumno dependiente. Hay espacios donde algunas veces el tema de la violencia ha salido como determinante social de la salud (...) No es algo que se busque de manera dirigida, entonces si al alumno se le ocurre sale el tema y se discute, pero es más bien oportunístico, no sistemático" (E1).

\section{Quehacer médico}

Si bien se reconoce que VIF-AS serían "temas médicos", su profundización y abordaje estaría sujeto a las consecuencias físicas o daños visibles presentes. 
"Yo creo que es un tema médico en la medida que afecta la salud de las personas, tanto psicológica como física." (FG 2).

Los entrevistados reconocen la importancia que los egresados de medicina logren detectar y reconocer "signos de alarma". También se menciona la relevancia de contar con flujos claros de trabajo, y la importancia de trabajar con profesionales de diversas áreas.

"un médico general debe ser capaz de sospechar violencia y tener al menos un flujograma de trabajo para ayudar a una persona que sufre violencia, a una familia que está pasando por esto. Creo que es parte del perfil de egreso" (E1).

\section{Competencias necesarias}

Todos los entrevistados mencionan la necesidad de contar con mayor capacitación en el tema, de manera de sentirse seguros a la hora de enseñar a sus estudiantes. En el dominio técnico surge la necesidad de reforzar conceptos teóricos y acceder a información más actualizada.

"yo creo que nosotros como médicos no fuimos formados para saber del tema. Probablemente lo que sabemos es lo que sale en los diarios y no es nuestra culpa, porque no es parte de la formación médica" (E1).

En relación a las competencias actitudinales, un aspecto aceptado por varios participantes tiene que ver con valorar el tema y asumirlo como algo que atañe al quehacer médico.

"quizás, la mayoría no se va a hacer cargo del tema, pero si saber dónde, en qué plazos, y a quién le vamos a hacer la derivación oportuna (...) Serían como los conocimientos mínimos que le podríamos pedir a uno de nuestros egresados" (FG2).

\section{Barreras y facilitadores}

Entre las principales barreras identificadas se encuentra el tiempo docente curricular, que se percibe por todos como una gran limitación. Los docentes identifican una amplia gama de contenidos relevantes para la formación del egresado, sin embargo, plantean poco tiempo dentro de sus cursos para agregar temas nuevos o darle la profundización necesaria.

"la principal barrera es el tiempo. La cantidad de cosas que como jefe de un curso de pregrado algún académico con algún interés en particular te pide incluir son varias (...)” (E2).

Esta falta de tiempo percibido también se vincula con la priorización que se realiza para decidir qué temas deben ser tratados.

"Finalmente, la gran barrera, al menos del punto de vista curricular con estos temas, tiene que ver con la ponderación. O sea, cuando tú tienes un montón de temas que son muy importantes, desde el punto de vista de prevalencia, de impacto en la calidad de vida de las personas, de los resultados de salud que tienen, entonces tú dices.... iya, prioricemos!, pero ¿cómo priorizamos? "(E1).

"A veces hay una pérdida como de sentido de la proporción y de la realidad. Me explico, nuestros alumnos son expertos en enfermedad de Crohn, sin embargo, pueden ser tremendamente ignorantes en cesación de tabaquismo" (FG2).

Otra de las barreras identificadas es la percepción de los docentes con relación a no tener los conocimientos necesarios para abordar VIF-AS, y a no visualizar a algún docente como el "responsable" o "referente del tema" para abogar por su incorporación formal en el currículo.

“¿cómo puedo yo poner dentro de la enseñanza del pregrado algunas habilidades de esta naturaleza? Tengo que crear un grupo de docentes de mi especialidad que se manejen en el tema. Ahi hay una primera barrera, porque el conocimiento es bajo." (E2).

Uno de los principales facilitadores mencionados es la visibilidad que la violencia de género ha tenido a nivel país durante el último año. Esto se reconoce como una oportunidad para instalar la discusión curricular sobre VIF-AS y para encontrarse con estudiantes más sensibilizados.

"Yo creo que el tema del contexto es un tremendo facilitador. Esto no era tema hace tres años (...) Ahora es el momento para decir que es algo que debemos enseñar, y nadie te va a decir que no" (E1).

"También hay un facilitador en lo que se produce en torno al tema. El tema no queda encapsulado, uno prende la tele y ve, por ejemplo, casos de femicidio o los problemas de abuso o maltrato que ha habido en algunas instituciones (...)" (FG2). 


\section{Recursos necesarios para un mejor abordaje}

Se observa común acuerdo sobre la necesidad de contar con capacitación específica en VIF-AS antes de poder enseñarlo. Se menciona que dentro de la escuela podrían existir algunos profesionales más capacitados (médicos familiares, pediatras, psiquiatras, psicólogos), que podrían asumir un rol de liderazgo.

"el tema tampoco estuvo presente, o muy poquito presente, en la formación de cada uno de nosotros, de modo que también tenemos ese lastre. Si se nos enseñó algo, se hizo de manera muy pequeña, un tanto superficial, como para poder dar una clase o hacer una actividad académica de nivel" (FG2).

La necesidad de contar con evidencia en relación al mejor abordaje posible de este tema, también resulta ser fundamental.

"Bueno, la especialidad nuestra se ha enseñado desde hace muchos años basada en evidencias, de modo que lo que nosotros requeriríamos es evidencia disponible. Desde cuáles son los beneficios de hacer un diagnóstico y tratamiento oportuno y cuáles son las herramientas para hacer eso" (E2).

\section{Discusión}

Las barreras identificadas para incorporar VIF-AS a los currículos de pregrado coinciden, en parte, con las reportadas por la literatura, destacando el tiempo curricular limitado y la priorización de contenidos. Llama la atención que pese a la altísima prevalencia de VIF-AS en el mundo y sus efectos conocidos y nocivos sobre la salud de las personas, estos temas aún no logran competir por un espacio formal en el currículo de un médico en formación.

A diferencia de lo reportado por la literatura, los docentes participantes reconocen que VIF-AS "son problemas de salud", y que su trato forma parte de la actividad clínica de un médico. En contraparte, sin embargo, se observa una tendencia a estimar que el abordaje de estos problemas desde el ámbito clínico y docente estaría focalizado en algunas especialidades (pediatría, medicina familiar, psiquiatría, ginecología-obstetricia), situación que se asume pero que no se corrobora, y que tampoco se apega a la realidad actual de nuestro programa de pregrado.

Si bien, que los docentes consideren que VIF-
AS son "problemas de salud" y que los médicos deben ser capaces de responder de manera competente supone una diferencia importante con lo reportado por la literatura, también destaca la creencia que su abordaje se concentra en "algunas especialidades". Este supuesto ha dejado el problema VIF-AS en manos de otros, "los especialistas en el tema", sin certeza de si esto efectivamente ocurre o de cuáles serían sus alcances.

Es difícil determinar si la declaración de los participantes sobre el rol que el médico tiene en VIF-AS se origina en una necesidad médica genuina, o como resultado del momento social en que nos encontramos con relación a violencia de género y malos tratos en la infancia. Evidentemente esto escapa a los alcances de este estudio, pero abre un punto de reflexión, y a su vez un primer desafío, ¿cómo integrar VIF-AS en las distintas especialidades y áreas de estudio?, pero de modo que se integre como un diagnóstico diferencial más y no sólo como una preocupación social, en un contexto en que el tema moviliza a la sociedad en su conjunto.

Un tema particularmente relevante para este equipo investigador es la percepción de los docentes con relación a no tener formación en VIF-AS, lo que les dificultaría poder enseñarlo. Este es un hecho que la literatura reconoce y que probablemente es real; los médicos, en general, entre ellos los docentes, tuvieron una escasa formación en VIF-AS, lo que puede dificultar que se perciban como responsables de la docencia, aun cuando puedan toparse con estos diagnósticos en sus actividades clínicas. La necesidad de contar con espacios de capacitación y trabajo guiados por parte de otros especialistas más involucrados en VIF-AS parece clave para los docentes, y es probable que las universidades deban intencionar esta estrategia, al menos en un comienzo.

Uno de los principales facilitadores para la inclusión curricular de VIF-AS es, a juicio de los participantes, el momento país que experimentamos con relación a violencia de género y la mayor sensibilidad que los estudiantes tienen al respecto.

VIF y AS recién comienzan a visibilizarse y problematizarse en su magnitud e implicancias a nivel social, y el mundo médico no puede quedarse atrás. La visibilidad que hoy tiene VIF-AS abre una oportunidad para gestionar cambios curriculares y avanzar en este desafío.

Existen distintas estrategias para reducir el 
impacto curricular de la inclusión de VIF-AS. Hay literatura extensa con relación al tema y parte de este equipo conduce un piloto en la Escuela de Medicina de la Pontificia Universidad Católica de Chile con estos fines. Este artículo espera contribuir en esta linea de desarrollo.

Agradecimientos: Departamento de Medicina Familiar de la Pontificia Universidad Católica de Chile, por el apoyo al equipo académico y el financiamiento del Fondo Intradepartamental 2015 de dicha institución.

\section{Referencias}

1. García-Moreno C, Jansen HA, Ellsberg M, Heise L, Watts CH; WHO Multi-country Study on Women's Health and Domestic Violence against Women Study Team. Prevalence of intimate partner violence: findings from the WHO multi-country study on women's health and domestic violence. Lancet 2006; 368 (9543): 1260-9.

2. Ministerio del Interior, Chile. Encuesta Nacional de Victimización por Violencia Intrafamiliar y Delitos Sexuales. 2008. Disponible en: http://estudios.sernam. $\mathrm{cl} /$ documentos/?eMTg1ODY1OQ==-Encuesta_Nacional_de_Victimizaci\%C3\%B3n_por_Violencia_Intrafamiliar_y_Delitos_sexuales,_2008. [Consultado el 10 de abril de 2017].

3. UNICEF. Larraín S, Bascuñán C. Cuarto estudio de Maltrato infantil UNICEF. 2011.

4. WHO multi-country study on women's health and domestic violence against women: summary report of initial results on prevalence, health outcomes and women's responses. Geneva, World Health Organization, 2005.

5. Institute of Medicine (US). Committee on the Training Needs of Health Professionals to Respond to Family
Violence, Board on Children, Youth, and Families. Cohn F, Salmon ME, Stobo JD, Editors. (2002). Confronting Chronic Neglect: The Education and Training of Health Professionals on Family Violence. National Academies Press (US), Washington, D.C.

6. Rojas P, Pinedo J. Violencia intrafamiliar y abuso sexual: ¿Cómo integrarlos al círculo de pregrado de medicina? ARS MEDICA Revista de Ciencias Médicas 2016; 41 (2): 54-61.

7. Gerber MR, Tan AK. Lifetime intimate partner violence exposure, attitudes and comfort among Canadian health professions students. BMC Research Notes 2009; 2, 191.

8. Hill JR. Teaching about family violence: a proposed model curriculum. Teach Learn Med 2005; 17 (2): 169-78.

9. Jamner MS, Stokols D. Promoting Human Wellness: New Frontiers for Research, Practice, and Policy. University of California Press, Berkeley (2000). Disponible en: https:// publishing.cdlib.org/ucpressebooks/view?docId=kt4r29q2tg;query=;brand=ucpress [Consultado el 11 de diciembre de 2017].

10. Miranda E, Garmendia F, Perales A, Mendoza P, Miano J, Calderón W. Evaluación de los planes curriculares y sílabos de las asignaturas de la Facultad de Medicina de la UNMSM, sobre la atención integral en salud a personas afectadas por la violencia. An Fac Med 2009; 70 (4): 273-6.

11. Narayan AP, Socolar RR, St Claire K. Pediatric residency training in child abuse and neglect in the United States. Pediatrics 2006; 117 (6): 2215-21.

12. Reece RM, Jenny C. Medical training in child maltreatment. Am J Prev Med 2005; 29 (5 Suppl 2): 266-71.

13. Dawn Miller, Chrystal Jaye; GPs' perception of their role in the identification and management of family violence. Fam Pract 2007; 24 (2): 95-101.

14. deLahunta EA, Tulsky A. Resistance to adding curricula about domestic violence. Acad Med 1998; 73: 726-7. 